\title{
Construction of maximally multiqubit entangled state by using CNOT gates Xin-wei Zha, Jun-ling Che
}

School of Science, Xi'an Institute of Posts and Telecommunications, Xi'an, 710121, P R China

\begin{abstract}
We propose a novel protocol to build a maximally entangled state based on controlled-not (CNOT) gates. In particular, we give detailed steps to construct maximally entangled state for 4-, 5-, and 6-qubit systems. The advantage of our method is the simple algebraic structure which can be realized via current experimental technology.
\end{abstract}

Keywords Maximally multiqubit entangled state; Bell-pair state; CNOT gates PACS numbers: 03.67.Hk, 03.65.Ud

\section{Introduction}

Entanglement is a key feature of quantum mechanical systems with wide applications to the field of quantum information theory. Multiqubit entangled states are regarded as a valuable resource for processing information. The class of quantum processes relying on entangled states include quantum state teleportation $^{1}$, quantum error correction ${ }^{2}$, quantum key distribution $(\mathrm{QKD})^{3}$.

A state has a large multipartite entanglement if its average bipartite entanglement is large. Maximally multi-qubit entangled states (MMES) ${ }^{4}$ are states whose entanglement is maximal for every (balanced) bipartition. It is proposed there that a MMES state in an n-qubit system must reach the maximal possible mixedness for each of its subsystems with $[n / 2]$ qubits $([x]$ takes the integer of $\mathrm{x})$. Therefore, we can determine whether a state is a MMES by whether a quantity representing the purity averaged over some $[n / 2]$-qubit subsystems of the state, defined as the averaged subsystem purity of the state, reaches its minimum ${ }^{4}$. We can obtain the averaged subsystem purity for a state as

$$
\pi_{M E}=\left(\begin{array}{c}
n \\
n_{A}
\end{array}\right)^{-1} \sum \pi_{A}
$$

where $n_{A}=[n / 2] ; \pi_{A}=\operatorname{Tr}_{A} \rho_{A}^{2}, \rho_{A}=\operatorname{Tr}_{\bar{A}}|\psi\rangle\langle\psi|, \quad \operatorname{Tr}_{X}$ denotes the partial trace over subsystem X. It isnobvious that for qubit cases $\frac{1}{N_{A}} \leq \pi_{M E} \leq 1$, where $N_{A}=2^{n_{A}}$. A maximally multi-qubit entangled state is a minimizer of $\pi_{M E}$.

For four-qubit systems, the criterion for the identification of maximally entangled four-qubit 
states had been presented ${ }^{5}$. It can be show that $\pi_{M E}=\frac{1}{3}$ for maximally entangled four-qubit state. The maximally entangled five-qubit state is discovered by D. K. Brown ${ }^{6}$. Using the numerical search procedure, Borras et al. ${ }^{7}$ found a kind of six-qubit maximally entangled state.

For five and six maximally entangled state qubits, it is also obtained $\pi_{M E}=\frac{1}{4}$ and $\pi_{M E}=\frac{1}{8}$, respectively. Recently, Zahra Raissi and Vahid Karimipour introduce a general method of gluing multi-partite states ${ }^{8}$. In this paper, we present a method for constructing maximally entangled states, generated by CNOT gates. The advantage of our approach is the simple algebraic structure which can be realized via current experimental technology.

\section{Construct Maximally Multipartite Entangled State}

Generally speaking, for small systems, maximally entangled quantum states are obtained easily. For example, for two-qubit state, the Bell state $|\varphi\rangle=\frac{1}{\sqrt{2}}(|00\rangle+|11\rangle)$ is maximally entangled; for three-qubit state, the GHZ state $|\varphi\rangle=\frac{1}{\sqrt{2}}(|000\rangle+|111\rangle)$ is also maximally entangled. However, the construction of maximally multipartite entangled quantum states is not as easy as the small systems. In this paper, the relation of maximally multipartite entangled quantum state with Bell state (or GHZ state) is discussed.

\subsection{Construct Maximally Entangled Four-qubit State via Cnot Gate}

For four-qubit, let us assume that prepared state is two Bell-pair state, that is

$$
|\psi\rangle_{1234}=\frac{1}{\sqrt{2}}(|00\rangle+|11\rangle)_{12} \frac{1}{\sqrt{2}}(|00\rangle+|11\rangle)_{34}
$$

In order to construct maximally four-qubit entangled state, we need to perform a set of CNOT gate on the prepared state.

Step 1 : First, we performs a CNOT gate on the two qubit 1and 4 with the qubit 1 as the controlling qubit. After this operation, the state of the four- qubit system becomes

$$
\left|\psi_{1}\right\rangle_{1234}=\frac{1}{2}(|0000\rangle+|0011\rangle+|1101\rangle+|1110\rangle)_{1234}
$$

Step 2 : We performs a CNOT gate on the two qubit 4 and 2 with the qubit 4 as the controlling qubit. 
After this operation, the state of the four- qubit system becomes

$$
\left|\psi_{2}\right\rangle_{1234}=C N_{42}\left|\psi_{1}\right\rangle_{1234}=\frac{1}{2}(|0000\rangle+|0111\rangle+|1001\rangle+|1110\rangle)_{1234}
$$

Step 3 : We performs a controlled phase gate on the two qubit 1 and 4 with the qubit 1 as the controlling qubit. The controlled phase gate is

$$
U_{c \varphi}=\left(\begin{array}{cccc}
1 & 0 & 0 & 0 \\
0 & 1 & 0 & 0 \\
0 & 0 & 1 & 0 \\
0 & 0 & 0 & -1
\end{array}\right)
$$

Then the state of the four- qubit system becomes

$$
\left|\psi_{3}\right\rangle_{1234}=U_{c \varphi(14)}\left|\psi_{2}\right\rangle_{1234}=\frac{1}{2}(|0000\rangle+|0111\rangle-|1001\rangle+|1110\rangle)_{1234}
$$

Step 4 :We performs Hadamard gate on the qubit 1, 2,3 the state of the four- qubit system becomes

$$
\begin{aligned}
& \left|\psi_{4}\right\rangle_{1234}=H_{1} \otimes H_{2} \otimes H_{3} \otimes\left|\psi_{3}\right\rangle_{1234} \\
& =\frac{1}{2 \sqrt{2}}(|0000\rangle-|0011\rangle-|0101\rangle+|0110\rangle+|1001\rangle+|1010\rangle+|1100\rangle+|1111\rangle)_{1234}
\end{aligned}
$$

This state is just the state of Yeo and Chua's maximally entangled four-qubit state ${ }^{9}$.

\subsection{Construct Maximally Entangled Five-qubit State via Cnot Gate}

For five-qubit, let prepared state is

$$
|\psi\rangle_{12345}=\frac{1}{\sqrt{2}}(|01\rangle+|10\rangle)_{12} \frac{1}{\sqrt{2}}(|001\rangle-|110\rangle)_{345}
$$

Step 1 : First, we performs a Hadamard gate on the qubit 1. After this operation, the state of the fivequbit system becomes

$$
\begin{aligned}
& \left|\psi_{1}\right\rangle_{12345}=H_{3}|\psi\rangle_{12345} \\
& =\frac{1}{2 \sqrt{2}}(|01\rangle+|10\rangle)_{12}(|001\rangle+|101\rangle-|010\rangle+|110\rangle)_{345}
\end{aligned}
$$


Step 2: We performs a CNOT gate on the two qubit 2 and 3 with the qubit 3 as the controlling qubit. After this operation, the state of the five- qubit system becomes

$$
\begin{aligned}
& \left|\psi_{2}\right\rangle_{12345}=C N_{32}\left|\psi_{1}\right\rangle_{12345} \\
& =\frac{1}{2 \sqrt{2}}\left[(|001\rangle-|010\rangle)_{345}(|01\rangle+|10\rangle)_{12}+(|101\rangle+|110\rangle)_{345}(|00\rangle+|11\rangle)_{12}\right]
\end{aligned}
$$

Step 3 : We performs a CNOT gate on the two qubit 1 and 5 with the qubit 1 as the controlling qubit. After this operation, the state of the five- qubit system becomes

$$
\begin{aligned}
& \left|\psi_{3}\right\rangle_{12345}=C N_{15}\left|\psi_{2}\right\rangle_{12345} \\
& =\frac{1}{2}\left[|010\rangle \frac{1}{\sqrt{2}}(|01\rangle-|10\rangle)+|100\rangle \frac{1}{\sqrt{2}}(|00\rangle-|11\rangle)\right. \\
& \left.\quad+|001\rangle \frac{1}{\sqrt{2}}(|01\rangle+|10\rangle)+|111\rangle \frac{1}{\sqrt{2}}(|00\rangle+|11\rangle)\right]_{12345}
\end{aligned}
$$

This state is just the state of maximally entangled five-qubit Brown state ${ }^{7}$.

\subsection{Construct maximally entangled six-qubit state via cnot gate}

For six-qubit, let prepared state is

$$
|\psi\rangle_{123456}=\frac{1}{\sqrt{2}}(|00\rangle+|11\rangle)_{12} \frac{1}{\sqrt{2}}(|01\rangle-|10\rangle)_{34} \frac{1}{\sqrt{2}}(|01\rangle+|10\rangle)_{56}
$$

Step 1 : First, we performs a CNOT gate on the two qubit 5 and 4 with the qubit 5 as the controlling qubit. After this operation, the state of the six-qubit system becomes

$$
\begin{aligned}
& \left|\psi_{1}\right\rangle_{123456}=C N_{54}|\psi\rangle_{123456} \\
& =\frac{1}{2 \sqrt{2}}(|00\rangle+|11\rangle)_{12}(|01\rangle-|10\rangle)_{34}|01\rangle_{56} \\
& +\frac{1}{2 \sqrt{2}}|10\rangle_{56}(|00\rangle+|11\rangle)_{12}(|00\rangle-|11\rangle)_{34}
\end{aligned}
$$

Step 2 : We performs a Hadamard gate on the qubit 5. the state of the six-qubit system becomes 


$$
\begin{aligned}
& \left|\psi_{2}\right\rangle_{123456}=H_{5}\left|\psi_{1}\right\rangle_{123456} \\
& =\frac{1}{4}\left[(|00\rangle+|11\rangle)_{12}(|01\rangle-|10\rangle)_{34}(|01\rangle+|11\rangle)_{56}\right. \\
& \left.+(|00\rangle+|11\rangle)_{12}(|00\rangle-|11\rangle)_{34}(|00\rangle-|10\rangle)_{56}\right]
\end{aligned}
$$

Step 3 : We performs a CNOT gate on the two qubit 4 and 1 with the qubit 4 as the controlling qubit. After this operation, the state of the six-qubit system becomes

$$
\begin{aligned}
& \left|\psi_{3}\right\rangle_{123456}=C N_{41}\left|\psi_{2}\right\rangle_{123456} \\
& =\frac{1}{4}\left[(-|00\rangle+|11\rangle)_{12}|10\rangle_{34}(|01\rangle+|11\rangle)_{56}+(|10\rangle+|01\rangle)_{12}|01\rangle_{34}(|01\rangle+|11\rangle)_{56}\right. \\
& \left.\quad+(|00\rangle+|11\rangle)_{12}|00\rangle_{34}(|00\rangle-|10\rangle)_{56}-(|10\rangle+|01\rangle)_{12}|11\rangle_{34}(|00\rangle-|10\rangle)_{56}\right]
\end{aligned}
$$

Step 4 : We performs CNOT gate on the two qubit 5 and 1 with the qubit 4 as the controlling qubit. After this operation, the state of the six-qubit system becomes

$$
\begin{aligned}
& \left|\psi_{4}\right\rangle_{123456}=C N_{51}\left|\psi_{3}\right\rangle_{123456} \\
& =\frac{1}{4}\left[(|00\rangle+|11\rangle)_{12}(|0000\rangle-|1001\rangle+|0111\rangle+|1110\rangle)_{3456}\right. \\
& \left.\quad+(|10\rangle+|01\rangle)_{12}(-|0010\rangle+|0101\rangle-|1011\rangle-|1100\rangle)_{3456}\right]
\end{aligned}
$$

Step 5 : We performs a controlled phase gate on the two qubit 2 and 3 with the qubit 2 as the controlling qubit. The controlled phase gate is

$$
U_{c \varphi}=\left(\begin{array}{cccc}
1 & 0 & 0 & 0 \\
0 & 1 & 0 & 0 \\
0 & 0 & 1 & 0 \\
0 & 0 & 0 & -1
\end{array}\right)
$$

Then the state of the four- qubit system becomes 


$$
\begin{aligned}
\left|\psi_{5}\right\rangle_{123456}=U_{c \varphi(23)}\left|\psi_{4}\right\rangle_{123456} \\
=\frac{1}{4}\left[|00\rangle_{12}(|0000\rangle-|1001\rangle+|0111\rangle+|1110\rangle)_{3456}\right. \\
\quad+|11\rangle_{12}(|0000\rangle+|1001\rangle+|0111\rangle-|1110\rangle)_{3456} \\
\quad+|10\rangle_{12}(-|0010\rangle+|0101\rangle-|1011\rangle-|1100\rangle)_{3456} \\
\left.\quad+|01\rangle_{12}(-|0010\rangle+|0101\rangle+|1011\rangle+|1100\rangle)_{3456}\right]
\end{aligned}
$$

Step 6: We performs CNOT gate on the two qubit 2 and 6 with the qubit 2 as the controlling qubit. After this operation, the state of the six-qubit system becomes

$\left|\psi_{6}\right\rangle_{123456}=C N_{26}\left|\psi_{5}\right\rangle_{123456}$

$=\frac{1}{4}\left[(|00\rangle)_{12}(|0000\rangle-|1001\rangle+|0111\rangle+|1110\rangle)_{3456}\right.$

$(|11\rangle)_{12}(|0001\rangle+|1000\rangle+|0110\rangle-|1111\rangle)_{3456}$

$+(|10\rangle)_{12}(-|0010\rangle+|0101\rangle-|1011\rangle-|1100\rangle)_{3456}$

$\left.+(|01\rangle)_{12}(-|0011\rangle+|0100\rangle+|1010\rangle+|1101\rangle)_{3456}\right]$

This state is just the state of six-qubit maximally entangled state, which is discovered by Borras et al ${ }^{8}$.

\section{Conclusions}

In summary, we have established maximally multipartite entangled states via Bell pair states, by using CNOT gate. Moreover, our method can also be extended to higher-dimensional quantum systems, which is one of the most important and necessary topics in the study of multipartite quantum entanglement. We hope that the scheme will be experimentally realized in the future.

\section{Acknowledgments}

This work was supported by the National Natural Science Foundation of China (61705182), National Science Foundation of Shannxi Province(2017JQ6024).

\section{References}

[1] C. H. Bennett, G. Brassard, C. Cr'epeau, R. Jozsa, A. Peres, and W. K. Wootters, Phys. Rev. Lett. 70, (1993) 1895.

[2]. R. Cleve, D.Gottesman and H. K. Lo, Phys. Rev. Lett. 83, (1999) 648.

[3]. A.K. Ekert, Phys. Rev. Lett. 67 (1991) 661; 
[4] P. Facchi, G. Florio, G. Parisi, S. Pascazio, Phys. Rev. A, 77 (2008) 060304

[5] X.W. Zha, H.Y. Song and and F. Feng, Commun.Theor.Phys. 56 (2011)827

[6] I. D. K.Brown, S. Stepney, A. Sudbery, and S. L. Braunstein, J. Phys. A Math. Gen. 38 (2005) 1119

[7] A.Borras,et al, J. Phys.A Math. Theor. 40(2007) 13407

[8] Z. Raissi, V. Karimipour, Quantum Inf. Process. 16 (2017)81

[9] Y. Yeo and W. K. Chua, Phys. Rev. Lett. 96 (2006) 060502. 